\title{
Lead Conductor Device
}

National Cancer Institute

\section{Source}

National Cancer Institute. Lead Conductor Device. NCI Thesaurus. Code C50028.

A bundle of leads designed to connect between a pacing device and implanted heart electrodes. 